\title{
Effects of extra dimensions on unitarity and the Higgs boson mass
}

\author{
Xiao-Gang $\mathrm{He}^{*}$ \\ Department of Physics, National Taiwan University, Taipei, Taiwan 10617, Republic of China
}

(Received 16 June 1999; published 12 January 2000)

\begin{abstract}
We study the unitarity constraint on two body Higgs boson elastic scattering in the presence of extra dimensions. The contributions from the exchange of spin- 2 and spin- 0 Kaluza-Klein states can have a large effect on the partial wave amplitude. The unitarity condition restricts the maximal allowed value for the ratio $r=\sqrt{s} / M_{S}$ of the center of mass energy $\sqrt{s}$ to the gravity scale $M_{S}$ to be less than one. Although the constraint on the standard Higgs boson mass for $r$ of order one is considerably relaxed, for small $r$ the constraint is similar to that in the standard model. The resulting bound on the Higgs boson mass is not dramatically altered if perturbative calculations are required to be valid up to the maximal allowed value for $r$.
\end{abstract}

PACS number(s): 11.80.Et, 11.10.Kk, 14.80.Bn

It has recently been proposed that gravitational effects can become large at a scale $M_{S}$ near the weak scale due to effects from extra dimensions [1,2], which is quite different from the traditional thought that gravitational effects only become large at the Planck scale $M_{P l}=1 / \sqrt{G_{N}} \approx 1.22 \times 10^{19} \mathrm{GeV}$. In this proposal the total space-time is $D=4+n$. The relation between the scale $M_{S}$ and the scale $M_{P l}$, assuming all extra dimensions are compactified with the same size $R$, is given by $M_{P l}^{2} \sim R^{n} M_{S}^{2+n}$. With $M_{S}$ near a TeV and $n=1, R$ would be too large. However, with $n$ larger than or equal to 2 , the theory is not ruled out for $M_{S} \sim 1 \mathrm{TeV}$. The lower bound for $M_{S}$ is constrained, typically, to be of order one $\mathrm{TeV}$ from present experimental data [3-7]. Future experiments will provide more stringent constraints [3-7]. There are many interesting phenomena due to the presence of extra dimensions [3-9]. In this paper we study effects from extra dimensions on the unitarity condition of partial wave amplitude in elastic two body Higgs boson scattering, and implications for the validity of perturbative calculations and for allowed Higgs boson mass.

In the minimal standard model (SM) there is a neutral Higgs boson $H$ resulting from spontaneous symmetry breaking of $\mathrm{SU}(2)_{L} \times \mathrm{U}(1)_{Y}$ to $\mathrm{U}(1)_{e m}$ due to the Higgs mechanism. The mechanism for spontaneous symmetry breaking is not well understood. There is no experimental evidence favoring any particular mechanism, such as the Higgs mechanism. The discovery of the Higgs boson and understanding of its properties are fundamentally important [10]. Many methods have been proposed to produce and to study the properties of Higgs bosons [10]. One of the most important issues is its mass. At present the lower bound on SM Higgs boson mass $m_{H}$ is set by the CERN $e^{+} e^{-}$collider LEP II to be $95.5 \mathrm{GeV}$ at $95 \%$ C.L. [11]. There are many theoretical constraints on the Higgs boson masses. The constraint from unitarity of partial wave amplitudes of longitudinal gauge boson and/or Higgs boson scatterings provide some of the interesting upper bounds on the mass [12-15].

In the presence of extra dimensions there are additional contributions to gauge and/or Higgs boson scatterings due to exchanges of spin-2 and spin-0 graviton excitations (the Kaluza-Kein states). The effects of these Kaluza-Klein (KK)

\footnotetext{
*Email address: hexg@phys.ntu.edu.tw
}

states can affect the partial wave amplitudes significantly if the ratio $r=\sqrt{s} / M_{S}$ of the center of mass frame energy $\sqrt{s}$ and the gravity scale $M_{S}$ is close to or larger than one. The unitarity condition for partial wave amplitude constrains $r$ to be less than one if perturbative calculations are valid. The allowed range for Higgs boson mass can also be different from that in the SM depending on the value of $r$. We find that effects from extra dimensions affect the Higgs boson scattering $\mathrm{HH} \rightarrow \mathrm{HH}$ the most. In the following we will concentrate on this process, and will comment on other processes at the end.

In the SM, the scattering amplitude for the process $H H$ $\rightarrow H H$ at the tree level is given by [13]

$$
M_{S M}(s, t)=3 \sqrt{2} G_{F} m_{H}^{2}\left(1+\frac{3 m_{H}^{2}}{s-m_{H}^{2}}+\frac{3 m_{H}^{2}}{t-m_{H}^{2}}+\frac{3 m_{H}^{2}}{u-m_{H}^{2}}\right)
$$

where $s, t$, and $u=4 m_{H}^{2}-s-t$ are the Mandelstam variables.

From the above expression, one obtains the $J=0$ partial wave amplitude [13]

$$
\begin{aligned}
a_{0}^{S M}= & \frac{1}{16 \pi}\left(\frac{4 p_{i} p_{f}}{s}\right)^{1 / 2} \frac{1}{s-4 m_{H}^{2}} \int_{-\left(s-4 m_{H}^{2}\right)}^{0} M_{S M}(s, t) d t \\
= & \frac{G_{F} m_{H}^{2}}{8 \sqrt{2} \pi} \sqrt{1-\frac{4 m_{H}^{2}}{s}}\left[3+\frac{9 m_{H}^{2}}{s-m_{H}^{2}}-\frac{18 m_{H}^{2}}{s-4 m_{H}^{2}} \ln \right. \\
& \left.\times\left(\frac{s}{m_{H}^{2}}-3\right)\right] .
\end{aligned}
$$

In the above, $p_{i, f}$ are the momentum of the initial and final Higgs boson in the center of mass frame, respectively.

The Higgs boson mass is constrained if one requires that the absolute value of $a_{0}$ does not violate the unitarity condition. There are many discussions of how to implement unitarity conditions [14]. For our purpose of demonstrating possible large effects of extra dimensions, we use a weak condition $\left|a_{0}\right|<1$ and work with the tree level amplitude to obtain a conservative bound. Applying this condition for $s$ $\gg m_{H}^{2}$, one obtains 


$$
m_{H}^{2}<\frac{8 \sqrt{2} \pi}{3 G_{F}}=1010 \mathrm{GeV} .
$$

If $m_{H}$ is substantially less than the above bound, the magnitude of the amplitude is well within the bound everywhere.

With extra dimensions, there are new contributions to $H H \rightarrow H H$ due to the exchange of KK states. Using the Feynman rules derived in Ref [6], we obtain

$$
\begin{aligned}
M_{N E W}(s, t)= & \frac{\kappa^{2}}{2}\left\{\frac{1}{s-m_{l}^{2}}\left[\left(2 m_{H}^{2}-t\right)^{2}+\left(2 m_{H}^{2}-u\right)^{2}-\frac{2}{3}\left(s-2 m_{H}^{2}\right)^{2}-\frac{4}{3} m_{H}^{2} s\right]+\frac{1}{t-m_{l}^{2}}\left[\left(2 m_{H}^{2}-s\right)^{2}+\left(2 m_{H}^{2}-u\right)^{2}\right.\right. \\
& \left.\left.-\frac{2}{3}\left(t-2 m_{H}^{2}\right)^{2}-\frac{4}{3} m_{H}^{2} t\right]+\frac{1}{u-m_{l}^{2}}\left[\left(2 m_{H}^{2}-s\right)^{2}+\left(2 m_{H}^{2}-t\right)^{2}-\frac{2}{3}\left(u-2 m_{H}^{2}\right)^{2}-\frac{4}{3} m_{H}^{2} u\right]\right\} \\
& +\kappa^{2}\left\{\frac{2(n-1)}{3(n+2)}\left(\frac{\left(s+2 m_{H}^{2}\right)^{2}}{s-m_{H}^{2}}+\frac{\left(t+2 m_{H}^{2}\right)^{2}}{t-m_{H}^{2}}+\frac{\left(u+2 m_{H}^{2}\right)^{2}}{u-m_{H}^{2}}\right)\right\} .
\end{aligned}
$$

The first and the second terms are due to exchanges of spin- 2 and spin-0 KK states, respectively.

Summing over all intermediate KK states and projecting out the $J=0$ partial wave amplitude, we obtain

$$
\begin{aligned}
a_{0}^{N E W}= & \sqrt{1-\frac{4 m_{H}^{2}}{s}}\left\{\frac{2}{3(n+2)}+\frac{11 s-12 m_{H}^{2}}{3 n M_{S}^{2}}-\frac{2}{3\left(s-4 m_{H}^{2}\right)}\left(M_{S}^{2} F n(4)+\left(6 s-8 m_{H}^{2}\right) F n(2)+\frac{6 s\left(s-4 m_{H}^{2}\right)+16 m_{H}^{2}}{M_{S}^{2}} F n(0)\right)\right\} \\
& +\frac{4(n-1)}{3(n+2)}\left\{\frac{1}{2}\left(s+2 m_{H}^{2}\right)^{2} \frac{s^{n / 2-1}}{M_{S}^{n+2}}\left[-i \pi+2 \operatorname{In}\left(M_{S} / \sqrt{s}\right)\right]+\frac{2}{n+2}+\frac{12 m_{H}^{2}-s}{n M_{S}^{2}}-\frac{2}{s-4 m_{H}^{2}}\left(M_{S}^{2} F n(4)+4 m_{H}^{2} F n(2)\right.\right. \\
& \left.\left.+\frac{4 m_{H}^{4}}{M_{S}^{2}} F n(0)\right)\right\} \sqrt{1-\frac{4 m_{H}^{2}}{s}}
\end{aligned}
$$

where

$\operatorname{In}(x)=\int_{0}^{x} \frac{y^{n-1}}{1-y^{2}} d y$,

$F n(\delta)=\int_{0}^{1} x^{n+\delta-1} \ln \left[\left(\frac{s-4 m_{H}^{2}}{M_{S}^{2}}+x^{2}\right) / x^{2}\right] d x$.

In the above we have used $\kappa^{2}=16 \pi G_{N}$ $=16 \pi(4 \pi)^{n / 2} \Gamma(n / 2) R^{-n} M_{S}^{-(n+2)}$ to relate $G_{N}$ to $M_{S}$.

In the expression for $a_{0}^{N E W}$ there are several constant terms which look dangerously large and are all canceled by terms proportional to $F n(4)$. In the large $M_{S}$ limit, $a_{0}^{N E W}$ is proportional to $1 / M_{S}^{4}$ and approaches zero as $M_{S}$ goes to infinity with $s$ and $m_{H}$ kept finite. In this limit the theory reduces to the SM. However, when the ratio $r=\sqrt{s} / M_{S}$ approaches one, the real and imaginary parts of $a_{0}^{N E W}$ both become of order one and can violate the unitarity condition $\left|a_{0}\right|<1$ even if $m_{H}$ is small. This indicates that the applicability of the effective theory, perturbatively, should be in the range $\sqrt{s}<M_{S}$. We remark that $a_{0}^{N E W}$ only becomes sensitive to $m_{H}$ for small $M_{S}$. With sufficiently large $M_{S}, a_{0}^{N E W}$ by itself does not provide interesting information for $m_{H}$. However since the SM is sensitive to Higgs boson mass, the total contribution will provide information about $m_{H}$. To have a better understanding of the details we consider $\left|a_{0}\right|$ as a function of $r=\sqrt{s} / M_{S}$ and $m_{H}$ for four typical cases: (a) $M_{S}=5 \mathrm{TeV}$ and $n=2 ;$ (b) $M_{S}=5 \mathrm{TeV}$ and $n=7$; (c) $M_{S}$ $=100 \mathrm{TeV}$ and $n=2$; and (d) $M_{S}=100 \mathrm{TeV}$ and $n=7$, for illustrations. For cases (c) and (d), the results reduce to the SM if $\sqrt{s}$ is not too close to $M_{S}$, while for cases (a) and (b) the effects from extra dimensions can be large. The results are shown in Figs. 1 and 2.

From Figs. 1 and 2 we see that in all cases where $r$ approaches one, $a_{0}$ becomes large and can violate the unitarity condition setting the perturbative range (the maximal allowed range $r_{\max }$ for $r$ where $\left|a_{0}\right|=1$ ) of the theory to be $0.81,0.96,0.81$, and 0.96 for cases (a), (b), (c), and (d) with $m_{H}=100 \mathrm{GeV}$, respectively. With larger $m_{H}$, the allowed range for $r_{\max }$ can be larger, as can be seen from Figs. 1 and 2 , due to the cancellation between contributions from the SM and extra dimensions, and the total $a_{0}$ is sensitive to $m_{H}$ as mentioned before. As long as $\sqrt{s}$ is much larger than $2 m_{H}$, $r_{\max }$ is not sensitive to $M_{S}$ but is sensitive to the number of extra dimensions $n$. We have studied different values for $n$ up to 7 and find that in all cases $r_{\max }$ is constrained to be less than one, but varies from case to case. Violation of the unitarity condition for $\sqrt{s}>M_{S}$ is not a big surprise because $M_{S}$ serves as a cutoff where gravity becomes strong. The calculation here provides a specific example. We stress, however, that results obtained using perturbative calculations with $\sqrt{s}$ close to $M_{S}$ are not reliable.

It has been shown [14] that in the SM at one loop level, for a given Higgs boson mass, there is a critical scale $\sqrt{s_{c}}$ 

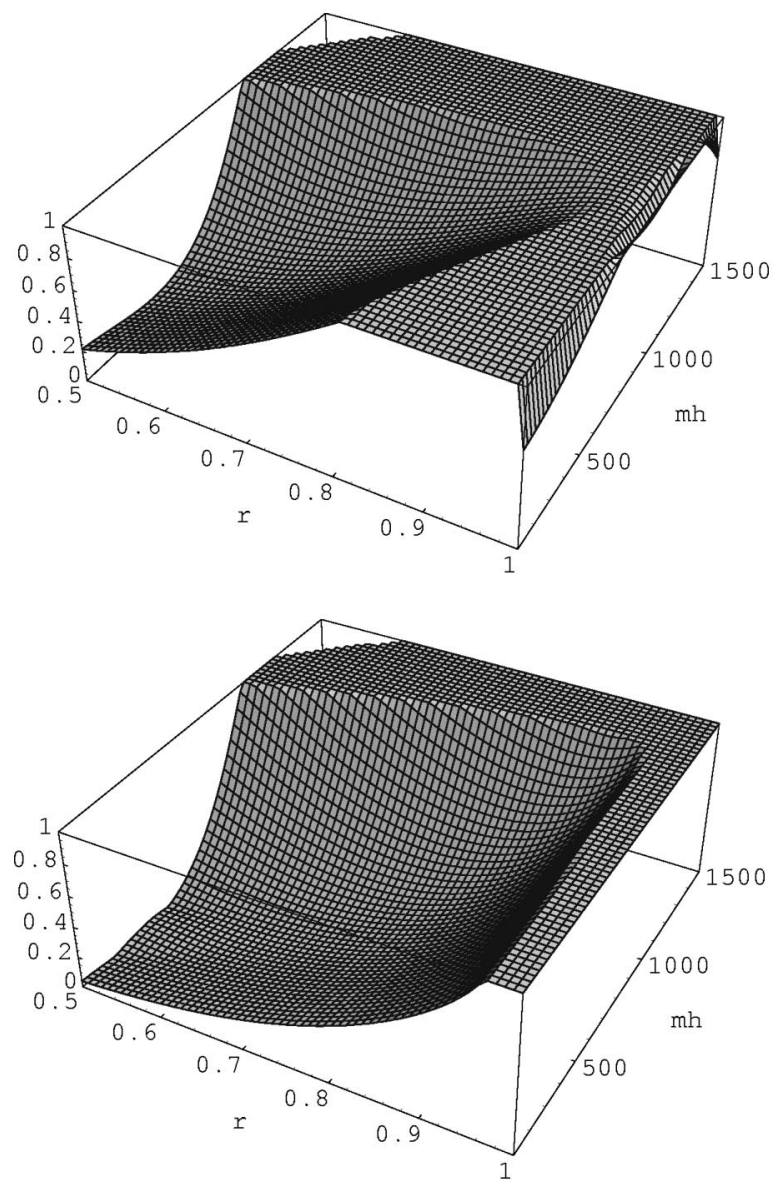

FIG. 1. $\left|a_{0}\right|$ (vertical axis) as a function of $r=\sqrt{s} / M_{S}$ and $m_{H}$ $(\mathrm{GeV})$ for $M_{S}=5 \mathrm{TeV}$ with $n=2$ and $n=7$, respectively. Cases (a) and (b) are shown at the top and bottom, respectively. The allowed parameter space with $\left|a_{0}\right|<1$ are located at the left-lower corners indicated by the dented regions.

and that when $s$ is larger than $s_{c}$, the unitarity condition is violated ( $s_{c}$ decreases as $m_{H}$ increases). With extra dimensions, we obtain the energy scale $s_{\max }$, below which the unitarity condition was reached earlier for small $M_{S}$ and $m_{H}$. If a stronger requirement on $\left|a_{0}\right|$ is made as discussed in Ref. [14], the allowed $r_{\max }$ will be smaller. The bound we obtained is a conservative one.

The amplitude $a_{0}$ is also sensitive to the Higgs boson mass $m_{H}$ as mentioned earlier. In all cases the allowed range for the Higgs boson masses is altered. In some cases the changes can be dramatic, as in case (c), in certain ranges of $r$. For small $r$ the bound on the Higgs boson mass is similiar to that in the SM. A Higgs boson mass bound with smaller $r$ is tighter than other ranges except for $r$ close to its maximal, as can be seen from Figs. 1 and 2. The bound on Higgs boson mass must be the smallest in the whole valid range of $r$ for perturbative calculation. Therefore the bound on the Higgs boson mass is not dramatically affected compared with the SM case, which is effectively determined with the small value for $r$ in our cases.

We also checked some other gauge and/or Higgs boson scattering processes. For example, the contribution to $\mathrm{HH}$
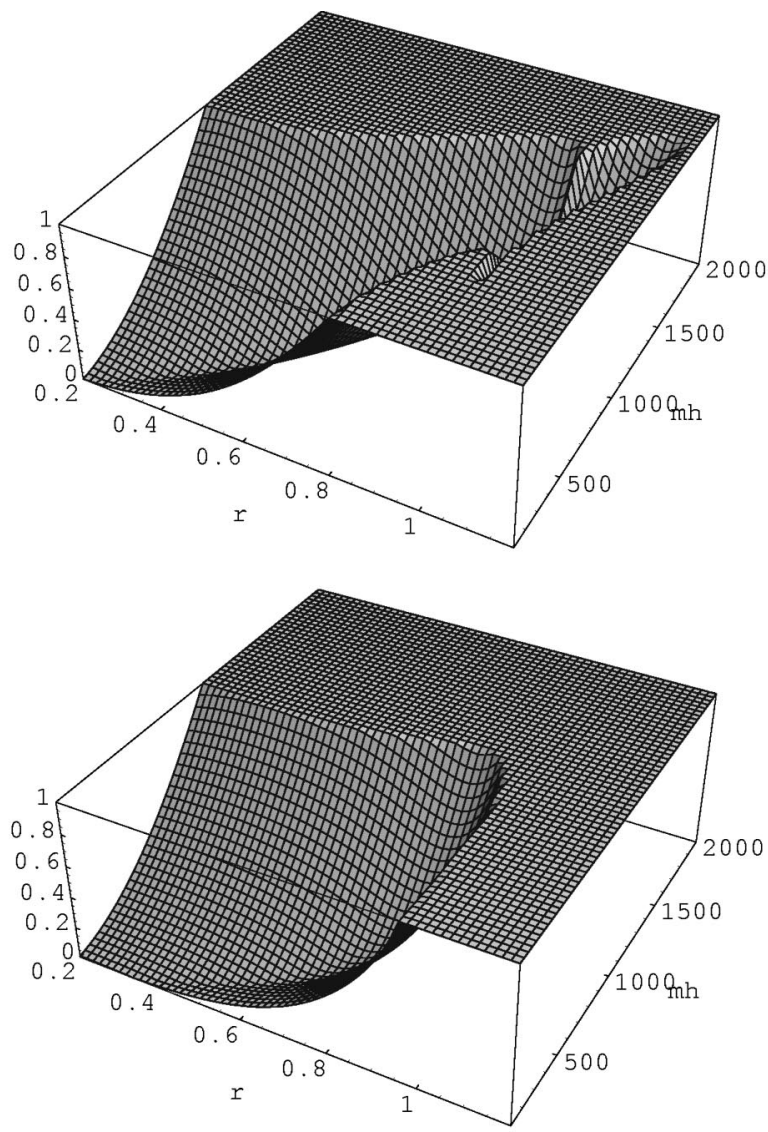

FIG. 2. The same as Fig. 1, but with $M_{S}=100 \mathrm{TeV}$. Cases (c) and (d) are shown at top and bottom, respectively. For $\sqrt{s}$ much smaller than $M_{S}(r \ll 1)$ the theory reduces to the SM.

$\rightarrow V V$ from extra dimensions in the limit neglecting the mass of the gauge boson $\mathrm{V}$ is given by

$$
\begin{aligned}
a_{0}^{N E W}(H H \rightarrow V V)= & \frac{1}{12} \frac{s^{n / 2-1}}{M_{S}^{n+2}\left[-i \pi+2 \operatorname{In}\left(M_{S} / \sqrt{s}\right)\right] s^{2}} \\
& \times\left(1-\frac{4 m_{H}^{2}}{s}\right)^{5 / 4} .
\end{aligned}
$$

This is smaller than that given for $H H \rightarrow H H$. In the unitarity consideration for $H H \rightarrow H H$ we obtain a stronger constraint.

In conclusion we have shown that effects from extra dimensions can have large contributions to $H H \rightarrow H H$. The range valid for perturbative calculations with extra dimensions is limited by the scale $M_{S}$. Results obtained with $\sqrt{s}$ $>M_{S}$ are not reliable. Although Higgs boson mass bound can be drastically affected for certain ranges of $\sqrt{s}$, the overall bound is not modified significantly if one requires the perturbative calculation for $H H \rightarrow H H$ to be valid up to the maximal allowed value of $r$.

This work is supported in part by the National Science Council of the Republic of China under Grant NSC 88-2112M-002-041. 
[1] N. Arkani-Hamed, S. Dimopoulos, and G. Dvali, Phys. Lett. B 429, 263 (1998); I. Antoniadis et al., ibid. 436, 257 (1998); N. Arhani-Hamed, S. Dimopoulos, and J. March-Russell, hep-ph/9809124.

[2] I. Antoniadis, Phys. Lett. B 246, 377 (1990); R. Sundrum, J. High Energy Phys. 07, 001 (1991); G. Shiu and S.-H. Tye, Phys. Rev. D 58, 106007 (1998); Z. Kakushadze and S.-H. Tye, Nucl. Phys. B548, 180 (1999); I. Antoniadis et al., Phys. Lett. B 436, 257 (1998).

[3] K.R. Dienes, E. Dudas, and T. Gherghetta, Phys. Lett. B 436, 55 (1998); Nucl. Phys. 537, 47 (1999); K.R. Dienes et al., ibid. B543, 387 (1999); N. Arkani-Hamed, S. Dimopoulos, and G. Dvali, Phys. Rev. D 59, 086004 (1999).

[4] G. Guidice, R. Rattazzi, and J. Wells, Nucl. Phys. B544, 3 (1999); E. Mirabelli, M. Perlestein, and M. Peskin, Phys. Rev. Lett. 82, 2236 (1999); J. Hewett, ibid. 82, 4765 (1999); P. Mathews, S. Raychaudhuri, and K. Sridhar, Phys. Lett. B 455, 115 (1999); 450, 343 (1999).

[5] T. Rizzo, Phys. Rev. D 59, 115010 (1999); hep-ph/9902273; Phys. Rev. D 60, 075001 (1999); K. Agashe and N. Deshpande, Phys. Lett. B 456, 60 (1999); K. Cheung and W.-Y. Keung, Phys. Rev. D 60, 112003 (1999); D. Atwood, S. BarShalom, and A. Soni, hep-ph/9903538; C. Balazs et al., Phys. Rev. Lett. 83, 2112 (1999); H. Goldberg, hep-ph/9904318; H. Davoudiasl, Phys. Rev. D 60, 084022 (1999); Kingman Cheung, Phys. Rev D 61, 015005 (2000); Phys. Lett. B 460, 383 (1999); A. Gupta, N. Mondal, and S. Raychaudhuri, hep-ph/9904234; G. Shiu, R. Shrock, and H. Tye, Phys. Lett. B 458, 274 (1999); K.-Y. Lee et al., Phys. Rev. D (to be published), hep-ph/9904355; 60, 093002 (1999); X.-G. He, ibid. 60, 115017 (1999); P. Mathews, P. Poulose, and K. Sridhar, hep-ph/9905395; I. Antoniadis, K. Benaki, and M. Quiros, hep-ph/9905311; T. Han, D. Rainwater, and D. Zeppenfeld, hep-ph/9905423.

[6] T. Han, J. Lykken, and R.-J. Zhang, Phys. Rev. D 59, 105006 (1999).
[7] M. Graesser, Phys. Rev. D (to be published), hep-ph/9902310; P. Nath and M. Yamaguchi, ibid. 60, 116004 (1999); M. Masip and A. Pomarol, ibid. 60, 096005 (1999).

[8] N. Arkani-Hamed and S. Dimopoulos, hep-ph/9811353; Z. Berezhiani and G. Dvali, Phys. Lett. B 450, 24 (1999); K. Dienes, E. Dudas, and T. Gherghetta, hep-ph/9811428; N. ArkaniHamed et al., hep-ph/9811448; A. Faraggi and M. Pospelov, Phys. Lett. B 458, 237 (1999); M. Sakamoto, M. Tachibana, and K. Takenaga, hep-ph/9902070; N. Arkani-Hamed and M. Schmaltz, Phys. Rev. D (to be published), hep-ph/9903417; G. Dvali and A. Smirnov, hep-ph/9904211; L. Hall and C. Kolda, Phys. Lett. B 459, 213 (1999); H.-C. Cheng, Phys. Rev. D 60, 075015 (1999); K. Yoshioka, hep-ph/9904433.

[9] S. Cullen and M. Perelstein, Phys. Rev. Lett. 83, 268 (1999); L. Hall and D. Smith, Phys. Rev. D 60, 085008 (1999); T. Banks, M. Dine, and A. Nelson, J. High Energy Phys. 06, 014 (1999); S. Cline, Phys. Rev. D (to be published), hep-ph/9904495; N. Arkani-Hamed et al., hep-ph/9903224; A. Riotto, hep-ph/9904485; M. Maggiore and A. Riotto, Nucl. Phys. B548, 427 (1999); A. Mazumbar, hep-ph/9902381; V. Barger et al., hep-ph/9905474.

[10] J. Gunion et al., Higgs Hunters' Guide (Addison-Wesley, Reading, MA, 1990); J. Gunion, A. Stange, and S. Willenbrock, in Electroweak Symmetry Breaking and New Physics at TEV, edited by T. L. Barklow, pp. 23-145, hep-ph/9602238.

[11] T. Greening, hep-ex/9903013.

[12] D. Dicus and V. Mathur, Phys. Rev. D 7, 3111 (1973).

[13] B. Lee, C. Quigg, and H. Tacker, Phys. Rev. D 16, 1519 (1977).

[14] S. Dawson and S. Willenbrock, Phys. Rev. Lett. 62, 1232 (1989); W. Marciano, G. Valencia, and S. Willenbrock, Phys. Rev. D 40, 1725 (1989); L. Durand, J. Johnson, and J. Lopez, Phys. Rev. Lett. 64, 1215 (1990); Phys. Rev. D 45, 3112 (1992); L. Durand, P. Maher, and K. Riesselmann, ibid. 48, 1084 (1993).

[15] E. Lendvai, G. Pocsik, and T. Torma, Mod. Phys. Lett. A 6, 1195 (1991); M. Seymour, Phys. Lett. B 354, 409 (1995). 